\title{
Recurrent host mobility in spatial epidemics: beyond reaction-diffusion
}

\author{
Vitaly Belik \\ Max Planck Institute for Dynamics and Self-Organization, Göttingen, Germany and \\ Department of Civil and Environmental Engineering, \\ Massachusetts Institute of Technology, Cambridge, MA, USA \\ Theo Geisel \\ Max Planck Institute for Dynamics and Self-Organization, Göttingen, Germany and \\ Department of Physics, University of Göttingen, Göttingen, Germany \\ Dirk Brockmann \\ Northwestern Institute on Complex Systems and \\ Department of Engineering Sciences and Applied Mathematics, \\ Northwestern University, Evanston, IL, USA
}

\begin{abstract}
Human mobility is a key factor in spatial disease dynamics and related phenomena. In computational models host mobility is typically modeled by diffusion in space or on metapolulation networks. Alternatively, an effective force of infection across distance has been introduced to capture spatial dispersal implicitly. Both approaches do not account for important aspects of natural human mobility, diffusion does not capture the high degree of predictability in natural human mobility patters, e.g. the high percentage of return movements to individuals' base location, the effective force of infection approach assumes immediate equilibrium with respect to dispersal. These conditions are typically not met in natural scenarios. We investigate an epidemiological model that explicitly captures natural individual mobility patterns. We systematically investigate generic dynamical features of the model on regular lattices as well as metapopulation networks and show that generally the model exhibits significant dynamical differences in comparison to ordinary diffusion and effective force of infection models. For instance, the natural human mobility model exhibits a saturation of wave front speeds and a novel type of invasion threshold that is a function of the return rate in mobility patterns. In the light of these new findings and with the availability of precise and pervasive data on human mobility our approach provides a framework for a more sophisticated modeling of spatial disease dynamics.
\end{abstract}




\section{INTRODUCTION}

The 2009 outbreak of a novel subtype (H1N1) of influenza A and its subsequent worldwide spread, the recent emergence of new human infectious diseases such as SARS in 2003, and the recurrent seasonal ourbreaks of influenza epidemics illustrate the growing importance of understanding the dynamics of human infectious diseases [1-3]. Key to understanding spatial dynamics in particular is an accurate assessments of human mobility patterns as infectious diseases spread among different locations due to movements of their host. Despite recent advances [4, 5] comprehensive data on mobility is typically unavailable, and modelers have to make reasonable assumptions when implementing host mobility in models. Often it is assumed that hosts move randomly (Fig 1 (a)) in the system yielding reactiondiffusion dynamics [2, 6-11]. An alternative heuristic approach captures spatial dynamics without explicitely accounting for host dispersal [12, 13]. Instead an effective force of infection between spatially separated populations mimics the effect of disease transmission across distance. Typically, this force is assumed to be proportional to the prevalence of the disease in one of the locations. Yet, because this approach lacks the explicit incorporation of host movements, a systematic analysis of conditions under which it is applicable is difficult.
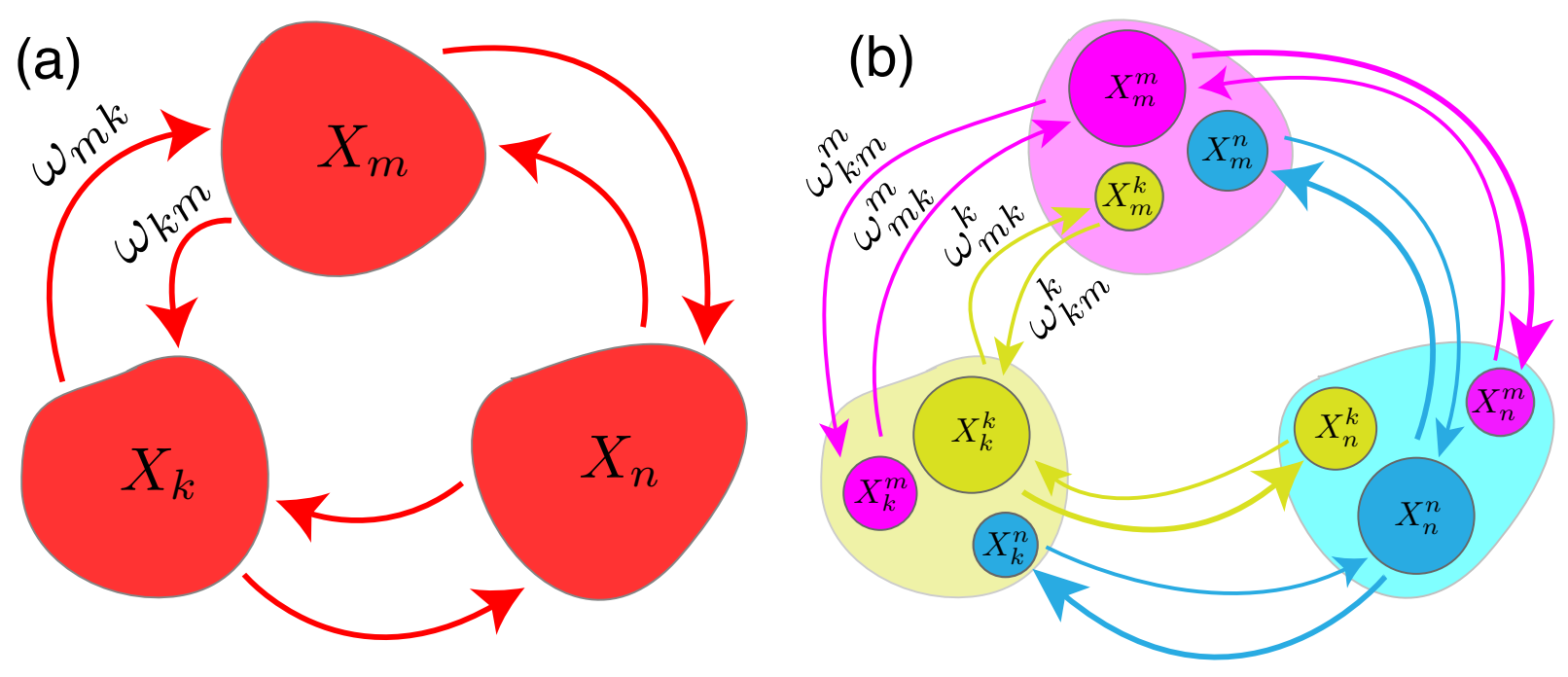

Figure 1: Models for human mobility: Patches and arrows represent individual populations and travel flux, respectively. (a) Diffusive dispersal: indistinguishable individuals travel randomly between different locations governed by the set of transition rates $\omega_{n m}$. (b) Natural human mobility: Individuals labeled $k$ travel from their base location $k$ to connected locations $m$ and back with travel rates $\omega_{m k}^{k}$ and $\omega_{k m}^{k}$, respectively.

Recently, human movement patterns became accessible based on pervasive mobility proxies 4, 5, 
14-[17]. One of the key findings of these studies confirmed the intuitive notion that humans spend most of their time in small sets of particular locations (home, work, shopping sites, etc.) and a person's mobility occurs predominantly between these individual-specific locations. Furthermore, a typical characteristic is the existence of one or two locations that function as an individuals base location, e.g. their homes to which individuals typically return before they travel to another place. A key feature of human mobility therefore is a bi-directional pattern in their trajectories among small sets of salient locations contrasting diffusion processes in which individual agents eventually visit every location in the entire system. Topologically, natural human mobility patterns can be described by individual mobility networks that possess a hub-and-spokes structure, in which a central hub represents a base location and a limited set of places connected by spokes the set of popular destination locations. Spreading phenomena across a large spatial scale occurs by virtue of interactions of agents that possess overlapping individual mobility networks.

In preceding papers and in this conference we have presented a stochastic model that explicitly accounts for the natural human mobility patterns described above [14, 18]. In particular, it respects the fact that individuals typically return to their unique base location before they travel to a new destination (recently a similar approach has been used by Balcan and Vespignani [19]). Here we investigate properties of this model and demonstrate that the dynamic consequences of natural mobility patterns are profound. Its basic aspects are depicted in Fig. 1. In this model mobility of the entire population is represented by a set of overlapping individual mobility networks. In the language of complex network theory each individual mobility pattern consists of a central node (the base location) connected to a set of accessible destinations (connected nodes) in the aforementioned hub-spokes topology.

Although mathematical metapopulation models have been proposed that are able to capture natural human mobility patterns [20, 21] it has remained elusive to what extent and under which conditions such models exhibit dynamic features that are qualitatively different from ordinary reaction-diffusion processes. It is unclear how these systems can be related to paradigmatic reaction-diffusion systems.

In the present article we show that the dynamics exhibits profound differences, indeed, as compared to ordinary reaction-diffusion systems. We concentrate on the analysis of epidemics on regular lattice and complex metapopulation networks. On lattices we obtain a generalization of the paradigmatic Fisher-Kolmogorov equation that describes wave propagation in reaction-diffusion systems. We show that the spatially continuous version of our model exhibits travelling wave solutions and compute their front velocities as a function of system parameters. Contrary to reaction-diffusion systems that exhibit a monotonic and unbounded increase of the front velocity with increasing travel rate, our model predicts an upper bound for front velocities. We show that the front shape strikingly differs from those predicted 
by reaction-diffusion systems and is more robust in response to changes in parameters. We introduce a commuting ratio parameter, a quantity present only in the natural mobility model, and investigate the front velocity as a function of it. Analysing a fully stochastic system in regular lattices as well as complex metapopulation networks we find that a global outbreak of a disease is determined by a novel threshold that is determined by the typical time spent away from individuals' base locations.

\section{NATURAL HUMAN MOBILITY AND DISEASE DYNAMICS}

\section{A. Disease Dynamics on a Metapopulation}

We consider a system of populations labeled $m=1, \ldots, M$ and assume that in each population an epidemic outbreak can be described by a compartmental SIR-model, i.e.

$$
I_{m}+S_{m} \stackrel{\alpha}{\rightarrow} 2 I_{m}, \quad I_{m} \stackrel{\beta}{\rightarrow} R_{m},
$$

in which the reactions govern infection due to the interaction of infected $(I)$ with susceptible $(S)$ individuals at rate $\alpha$, and recovery of an infected individuals at rate $\beta$, respectively. The number of individuals in a population is given by $N_{m}=S_{m}+I_{m}+R_{m}$. The spread of an epidemic across the set of $M$ populations is governed by the exchange of individuals between populations. The most prominent and conceptually clearest ansatz is diffusive dispersal between populations in which individuals of each class move from location $n$ to $m$ at rate $\omega_{m n}$, i.e

$$
X_{n} \stackrel{\omega_{m n}}{\longrightarrow} X_{m}
$$

where $X_{n}$ represents $I_{n}, S_{n}$ or $R_{n}$. The rates $\omega_{m n}$ generate an equilibrium distribution $\bar{N}_{n}$ of individuals among $M$ populations. Assuming that for a pair of populations exchange rates are nonzero, detailed balance is fullfilled, i.e. $\bar{N}_{n} / \bar{N}_{m}=\omega_{n m} / \omega_{m n}$. In the following we will assume that the system is in equilibrium with respect to dispersal, i.e. $N_{m}=\bar{N}_{m}$, yielding the following mean-field dynamical equations:

$$
\begin{aligned}
\partial_{t} I_{n} & =\alpha S_{n} I_{n} / \bar{N}_{n}-\beta I_{n}+\sum_{m \neq n}\left(\omega_{n m} I_{m}-\omega_{m n} I_{n}\right) \\
\partial_{t} S_{n} & =-\alpha S_{n} I_{n} / \bar{N}_{n}+\sum_{m \neq n}\left(\omega_{n m} S_{m}-\omega_{m n} S_{n}\right) \\
R_{n} & =\bar{N}_{n}-S_{n}-I_{n} .
\end{aligned}
$$

Note that in the reaction-diffusion system individuals are indistinguishable apart from their infection status and move about randomly between all available locations $m$. This approach has been employed both in complex networks of coupled populations as well as simplified lattice models [11,22]. 
The relation to spatially continuous models is best illustrated in a system of linearly aligned populations separated by distance $l$, at locations $x=n l$, uniform population size $\bar{N}_{n}=\bar{N}=$ const. and travel between neighboring populations only, i.e. $\omega_{n m}=\omega \delta_{n-1, m}+\omega \delta_{n+1, m}$. The overall uniform rate $\omega$ is related to the waiting time in a given location $\tau=\omega^{-1}$. In the limit $l, \tau \rightarrow 0$ with $D=l^{2} / \tau$ this model yields the 1-d reaction-diffusion system

$$
\begin{aligned}
& \partial_{t} j=\alpha j s-\beta j+D \partial_{x}^{2} j \\
& \partial_{t} s=-\alpha j s+D \partial_{x}^{2} s
\end{aligned}
$$

where $j(x, t) l=I_{n} / \bar{N}_{n}$ and $s(x, t) l=S_{n} / \bar{N}_{n}$. These equations are related to the Fisher-Kolmogorov equation [8,9]. For sufficiently localized initial conditions $j(x, t=0)$ this system exhibits travelling waves with front velocity

$$
c=2 l \sqrt{(\alpha-\beta) / \tau} \sim \sqrt{\omega},
$$

that monotonically increases with the global mobility rate $\omega$.

In order to account for individual mobility networks that exhibit base locations and natural recurrent movements we propose the following generalization of Eqs. (1), (2): We assume that individuals can be grouped into subpopulations defined by two indices, $n$ and $k$. The first index determines the current location $n$, the second the base location $k$. Generally, the dispersal dynamics is then governed by a set of reactions:

$$
X_{n}^{k} \underset{\omega_{n m}^{k}}{\stackrel{\omega_{m n}^{k}}{\rightleftarrows}} X_{m}^{k}
$$

This implies that individuals of class $k$ possess their specific dispersal rate matrix $\omega_{m n}^{k}$ that is conditioned on the base location $k$. The rate $\omega_{m n}^{k}$ determines how individuals of type $k$ travel from location $n$ to $m$, for fixed $k$ the matrix $\omega_{m n}^{k}$ represents the aforementioned individual mobility network for individuals of type $k$. The dynamical system, incorporating disease dynamics, is given by

$$
\begin{aligned}
\partial_{t} I_{n}^{k} & =\frac{\alpha}{N_{n}} S_{n}^{k} \sum_{m} I_{n}^{m}-\beta I_{n}^{k}+\sum_{m}\left(\omega_{n m}^{k} I_{m}^{k}-\omega_{m n}^{k} I_{m}^{k}\right) \\
\partial_{t} S_{n}^{k} & =-\frac{\alpha}{N_{n}} S_{n}^{k} \sum_{m} I_{n}^{m}+\sum_{m}\left(\omega_{n m}^{k} S_{m}^{k}-\omega_{m n}^{k} S_{m}^{k}\right),
\end{aligned}
$$

where $I_{n}^{k}$ and $S_{n}^{k}$ are the number of infecteds and of susceptibles of type $k$ located at $n$, respectively. $N_{n}$ denotes the total number of individuals in location $n$, i.e. $N_{n}=\sum_{k}\left(I_{n}^{k}+S_{n}^{k}+R_{n}^{k}\right)$. Note that if the rates $\omega_{n m}^{k}$ are independent of $k$, we recover the ordinary reaction-diffusion case.

In the following we consider the case of overlapping hub-spokes networks corresponding to commuting between base and destination locations. This imposes restrictions on the rates $\omega_{n m}^{k}$, it implies 
that $\omega_{n m}^{k}=0$ if $k \neq n$ and $k \neq m$. That means individuals of type $k$ that are located at $m$ cannot travel to $n$ without returning to $k$ first. We further assume that $\omega_{m k}^{k}=\omega^{-}$, i.e. the return rate is uniform for all $k$ and $m$. This assumption implies that individuals typically spent the same amount of time in distant locations before returning to their base. Assuming that travel of the entire system is equilibrated, we obtain

$$
\bar{N}_{n}=\sum_{k} \frac{\delta_{n k}+\left(1-\delta_{n k}\right) \omega_{k n}^{k} / \omega_{n k}^{k}}{1+\sum_{m \neq k} \omega_{m k}^{k} / \omega_{k m}^{k}} N^{k},
$$

where $\bar{N}_{n}$ is the stationary number of individuals located in population $n$ and $N^{k}=\sum_{n} N_{n}^{k}$ is the total number of individuals of type $k$ (i.e. they belong to base location $k$ ).

An important limiting case is a situation in which mobility rates are large compared to the rates associated with the infection and recovery dynamics, i.e. $\omega_{m k}^{k}, \omega^{-} \gg \alpha, \beta$. In this case detailed balance is fulfilled for infecteds and susceptibles separately and the last terms in Eq. 7) vanish. If we assume that $\omega_{m k}^{k} / \omega^{-} \ll 1$ which implies that individuals belonging to $k$ remain at their base most of time, Eq. (7) can be reduced to the effective force of infection model [12]:

$$
\frac{d}{d t} I^{k}=\alpha S^{k} \sum_{m} \epsilon_{k m} I^{m}-\beta I^{k},
$$

where $I^{k}=\sum_{m} I_{m}^{k}$ is the number of infected individuals belonging to location $k$ and coupling strengths $\epsilon_{n k}=\sum_{m} p_{n}^{m} p_{k}^{m} / \bar{N}^{m}$ are explicitly related to travel rates and $p_{n}^{m}=\bar{N}_{n}^{m} / \bar{N}_{n}$ is the occupation probability. Hence direct coupling represents a special case of our model, see also [21].

In order to investigate the dynamic consequences of natural human mobility patterns as captured by Eqs. 77, we consider a system analogous to the one-dimensional spatially homogeneous system leading to the reaction-diffusion Eq. (4). We consider a 1-d lattice of populations of size $N$ separated by a distance $l$, assume next-neighbor coupling and allow only infecteds to travel (relaxing this restriction does not change the main results but eases the analysis). We denote the number of infecteds at their base location by $I_{n}^{n}$ and the number of infecteds at neighboring locations $(n-1)$ and $(n+1)$ by $I_{n}^{-}$or $I_{n}^{+}$, respectively. This yields

$$
\begin{aligned}
I_{n}^{n}+S_{n} & \stackrel{\alpha}{\rightarrow} 2 I_{n}^{n} \\
I_{n \mp 1}^{ \pm}+S_{n} & \stackrel{\alpha}{\rightarrow} I_{n \mp 1}^{ \pm}+I_{n}^{n} \\
I_{n}^{n} & \stackrel{\omega^{+}}{\underset{\omega^{-}}{\rightleftharpoons}} I_{n}^{ \pm}
\end{aligned}
$$

where $S_{n}$ denotes the number of susceptibles at $n . \omega^{+}$and $\omega^{-}$denote forward and return rates, respectively. In the corresponding dynamical system we can approximate $S_{n}, I_{n}^{ \pm}$by their continuous counterparts: $I_{n \pm 1}^{ \pm} \rightarrow I^{ \pm}(x \pm l) \approx I^{ \pm} \pm l \nabla I^{ \pm}+\frac{l^{2}}{2} \Delta I^{ \pm}$. In an equilibrated homogeneous lattice the size 

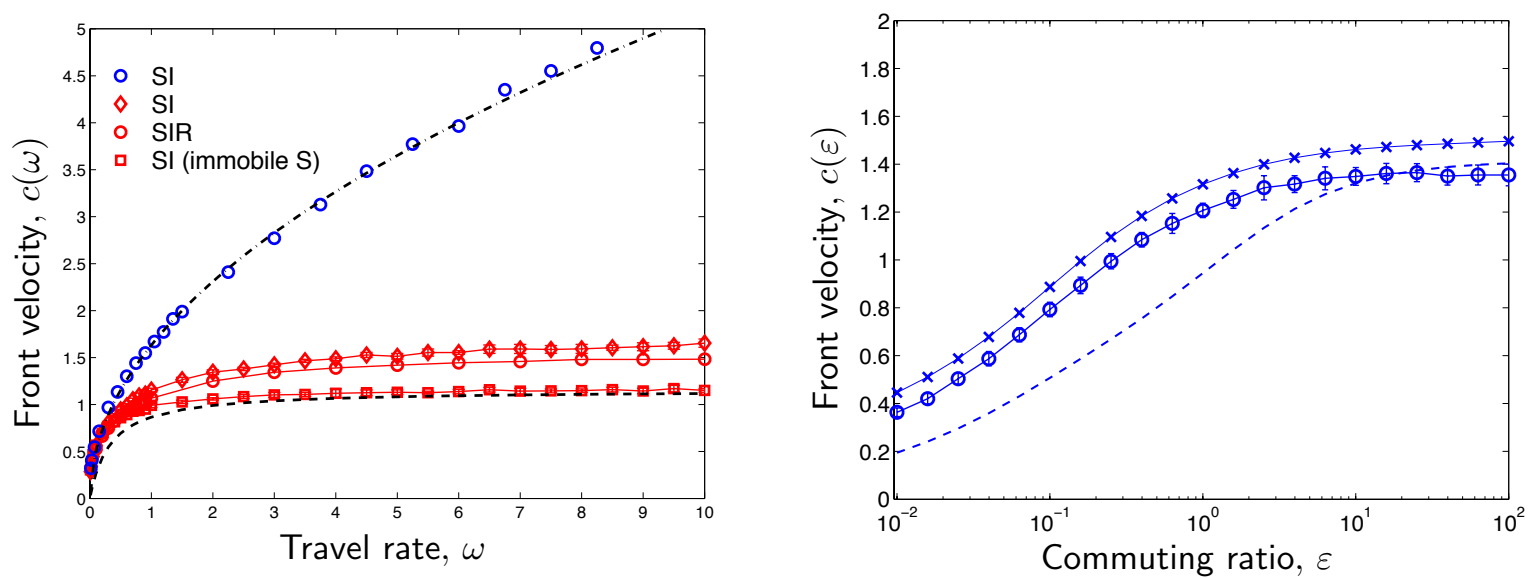

Figure 2: Front propagation in natural human mobility models. Left: Front velocity $c(\omega)$ as a function of travel rate $\omega$ for a reaction-diffusion and the natural mobility model. Numerical results of the stochastic simulations with $N=10^{4}$ agents per site are depicted by symbols for reaction-diffusion (blue) and natural mobility (red) models. Analytical results for the reaction-diffusion system, Eqs. (5) and natural mobility model, Eqs. (11) are depicted by dash-dotted and dashed lines, respectively. Right: Front velocity $c(\varepsilon)$ as a function of the commuter ratio $\varepsilon$ (for $\omega=1$ ). The dashed curve illustrates the analytical result, Eq. (15). Crosses and circles depict results of numerical solutions of the mean-field equations 7 and stochastic simulations of the natural mobility in a spatial SI model, respectively.

of a single population remains constant during an epidemic. Introducing concentrations $u_{n}=I_{n} / N$, $v_{n}=\left(I_{n}^{+}+I_{n}^{-}\right) / N$ and $w_{n}=\left(I_{n}^{+}-I_{n}^{-}\right) / N$ this yields:

$$
\begin{aligned}
\partial_{t} u & =\alpha(1-u-v)(u+v+D \Delta v+l \nabla w)+\omega^{-} v-2 \omega^{+} u \\
\partial_{t} v & =2 \omega^{+} u-\omega^{-} v \\
\partial_{t} w & =-\omega^{-} w
\end{aligned}
$$

where $D=l^{2} / 2$. The third equation is solved by $w \sim e^{-\omega^{-} t}$ and for $t \gg 1 / \omega^{-}$yields $w \approx 0$. We can therefore discard $w$ leaving only the first two equations in 10 . Steady states are $\bar{u}=0, \bar{v}=0$ and $\bar{u}=$ $\omega^{-} /\left(2 \omega^{+}+\omega^{-}\right), \bar{v}=2 \omega^{+} /\left(2 \omega^{+}+\omega^{-}\right)$. In the non-zero steady state concentration of infecteds in one city is given by $u+v=1$. In order to compare this system to the reaction-diffusion model, we calibrate both systems such as to keep the total flux of individuals between two particular locations equal in both systems. 


\section{RESULTS}

In epidemiology key questions concern conditions under which an epidemic can spread. In this case it is generally the first task to compute or estimate the speed at which an epidemic proliferates throughout the entire system. On regular lattices with dispersal only among adjacent site this task is equivalent to computing asymptotic wavefront speeds. In complex network topologies alternative quantities are useful, e.g. the time to reach the epidemic peak. Below, we investigate the velocity of epidemic propagation on a lattice, and subsequently provide results in a complex network topology.

\section{A. Front velocity in lattice systems}

Using the traveling wave ansatz $f(x, t)=g(x-c t)$ for $u$ and $v$, performing a linear stability analysis of the disease-free state, we find that the system defined by Eqs. (10) exhibits traveling wave solutions with front velocity given by

$$
c=\frac{2 \alpha \omega^{+} \sqrt{D\left(2+\frac{\omega^{-}}{\omega^{+}}\right)}}{\alpha+\omega^{-}+2 \omega^{+}} .
$$

If the forward and return rates $\omega^{+}$and $\omega^{-}$are significantly different, two extreme cases can be considered. In the limit $\omega^{+} \rightarrow 0$ we find $c \rightarrow 0$ as expected, i.e. no propagation can be sustained in the limit of individuals not leaving their base. If the backward rate $\omega^{-}$is small, the system is determined exclusively by the forward rate $\omega^{+}$.

It is instructive to first consider a balanced system, i.e. $\omega^{+}=\omega^{-}=\omega$. In this case Eq. 111) simplifies to

$$
c=\frac{2 \sqrt{6 D} \alpha \omega}{\alpha+3 \omega} .
$$

The front velocity as a function of infection rate $\alpha$ and travel rate $\omega$ as well as results of stochastic numerical simulations are depicted in Fig. 2. For comparison, the front velocity of the reaction-diffusion scenario with the same global travel rate $\omega$ is depicted as well. The velocity in this case is given by Eq. (5) which increases with travel rate according to $\sim \sqrt{\omega}$. In contrast, the natural mobility model exhibits a saturation of the front velocity with increasing travel rate. From Eq. (12) it follows that the asymptotic value of the velocity is proportional to the reaction rate $\lim _{\omega \rightarrow \infty} c=2 \alpha \sqrt{2 D / 3}$.

The existence of a saturation is a consequence of natural mobility patterns that are restricted to individual mobility networks. Likewise the unbounded increase in front velocity in a reaction-diffusion systems is a consequence of the unnatural assumption that increasing the travel rate also increases an individual's access to the entire system. In the more realistic natural mobility model increasing $\omega$ only 
increases the rate of travel between the base and the two neighboring sites and does not imply faster coverage of the entire system.

In contrast to the reaction-diffusion system with only one rate parameter $\omega$, the natural human mobility model possesses two travel rates, $\omega^{+}$and $\omega^{-}$. The total flux between two neighboring locations is given by $F_{n m}=\omega^{+} N_{n}^{n}+\omega^{-} N_{m}^{n}$, for $m=n \pm 1$. In equilibrium, detailed flux balance requires $F_{n m}=F_{m n}$. Comparing to the reaction-diffusion system, the total flux is given by $F_{n m}^{0}=\omega N$. In order to compare the dynamics of both systems quantitatively it is plausible to gauge both systems such that flux is identical, i.e.

$$
\omega^{+} N_{n}^{n}+\omega^{-} N_{m}^{n}=\omega N
$$

To simplify the analysis it is convenient to introduce a commuting ratio $\varepsilon=\omega^{+} / \omega^{-}$. In a situation in which individuals dwell at their base location most of the time, the commuting ratio is a small, $\varepsilon \ll 1$. We can express forward and return rates in terms of the global travel rate $\omega$ and the commuting ratio $\varepsilon$ according to:

$$
\omega^{+}=\frac{1+2 \varepsilon}{2} \omega \quad \text { and } \quad \omega^{-}=\frac{1+2 \varepsilon}{2 \varepsilon} \omega
$$

With these definitions expression (11) can be rewritten as

$$
c=\frac{2 \alpha \varepsilon \sqrt{2 D(2+1 / \varepsilon)}}{1+2 \varepsilon+2 \alpha \varepsilon(1+2 \varepsilon)^{-1} \omega^{-1}} .
$$

Fig. 3 depicts the front velocity as a function of the commuting ratio at the fixed travel rate $(\omega=1)$ and illustrates a significant change of $c$ as a function of $\varepsilon$. Since the commuting ratio is not defined for ordinary reaction-diffusion systems, this effect cannot be captured in these systems.

A surprising result in the behavior of the front velocity for very low travel rates is depicted in Fig. 2 which illustrates a noticeable deviation of the stochastic system from both, the analytical prediction of Eq. (11) and the results of the numerical solution to Eqs. (7). The deviation of the numerical solution from the Monte Carlo simulations for small $\omega$ is due to the finite number of agents per site. We observe a crossover from a linear scaling with $\omega$ (symbols) towards the numerical mean-field solution (solid blue line). The regime of low travel rates effectively corresponds to high infection rates $(\alpha \gg \omega)$. This implies that an outbreak takes place almost instantaneously in a neighboring location and and epidemic essentially jumps from one location to the next. The rate of hopping is proportional to $N \omega$, i.e. to the flux of individuals between locations, where $N$ is a typical of individuals per site. The crossover from discrete to continuous behavior occurs when $\alpha \sim \omega N$, i.e. $\omega_{\mathrm{c}} \sim \alpha / N$. Note that the slow convergence 


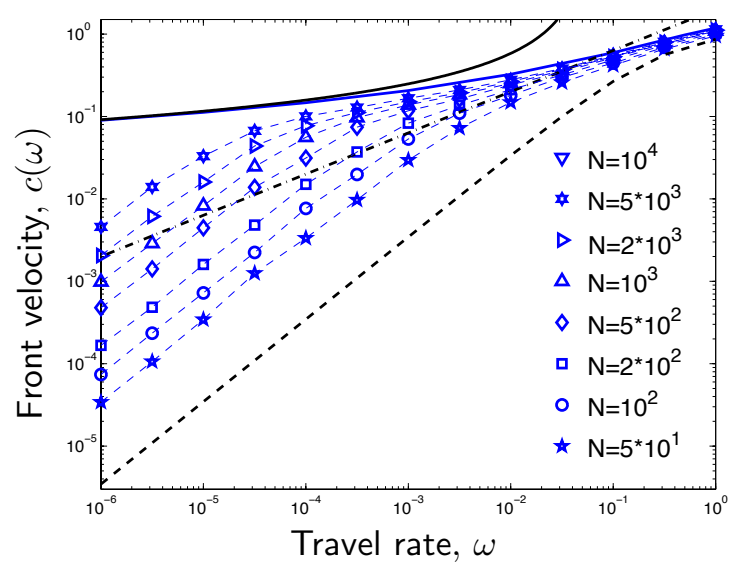

Figure 3: Front speed in the limit of small travel rates. Bottom Left: Front velocity $c(\omega)$ in the regime of low travel rates $\omega$. Symbols reflect stochastic simulations for different values of particles per site. Dashed and dashdotted lines represent analytical results given by Eqs. [11] and [5, respectively. Solid blue line corresponds to the numerical solution of Eqs. (7) and the solid black line represents the analytical result of Eq. (19).

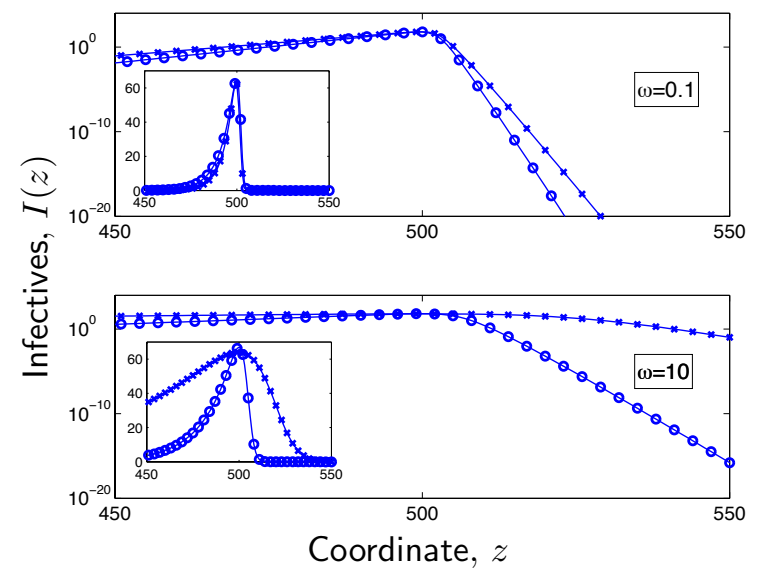

Figure 4: Front shape at the leading edge: Front shape for different global travel rates $\omega$ in the reaction-diffusion system (crosses) and natural mobility model (circles) combined with local SIR dynamics.

of the velocity towards zero with decreasing travel rate can be understood qualitatively: Let's consider just two locations with agents that can travel between them. Without loss of generality we consider an SI epidemic. At the beginning of the epidemic the number of infecteds in the second location is small and we can linearize the standart SI dynamics for the second population, i.e.

$$
\frac{d j_{2}}{d t} \approx \alpha j_{2}+\omega j_{1}
$$

where we neglect the backward flux of the individuals from the second location. Integrating Eq.16 by means of the integrating factor $j_{2}(t)=e^{\alpha t} \int_{0}^{t} d \tau j_{1}(\tau)$ and using the solution of the SI model for the first 

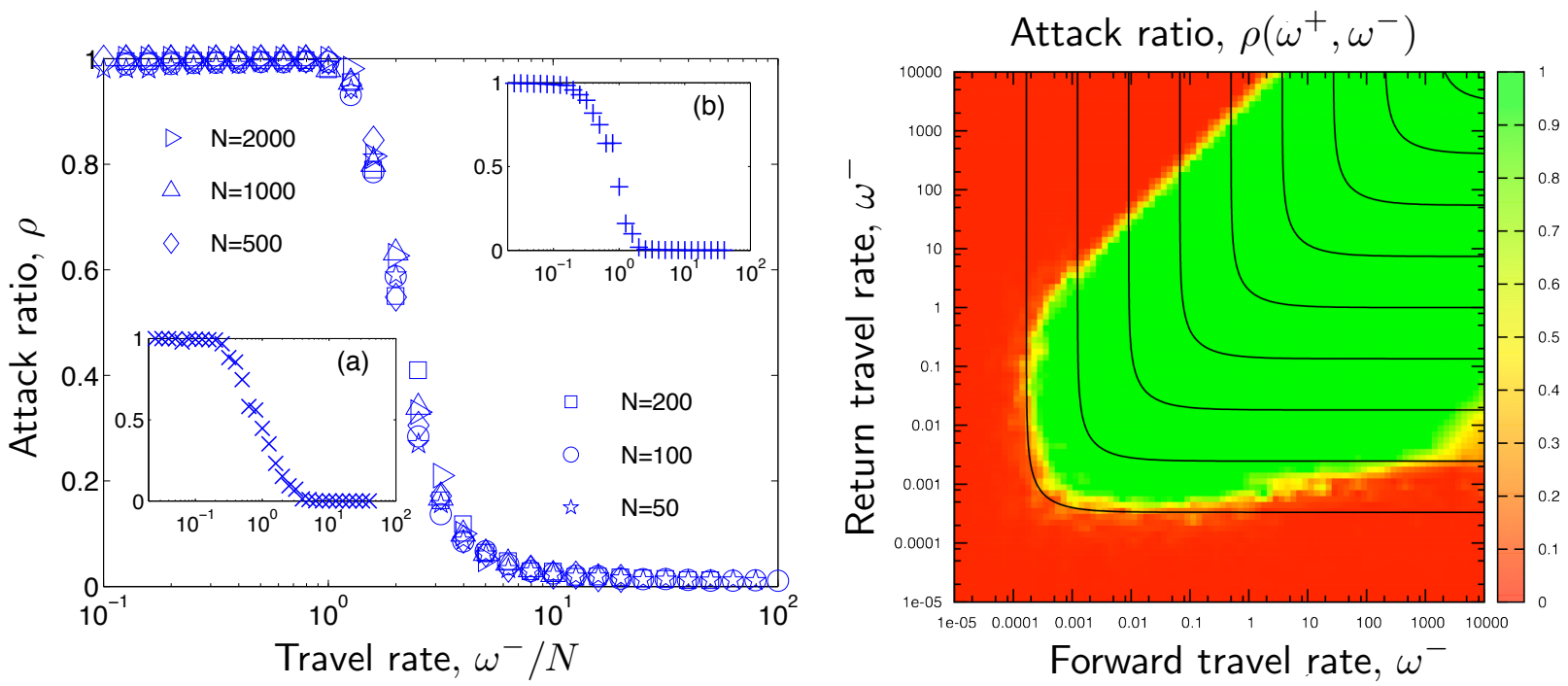

Figure 5: A novel type of invasion threshold. Left: The panel depicts the results of stochastic simulations of an SIR epidemic in combination with natural mobility patterns. The attack ratio $\rho\left(\omega^{-}\right)$as a function of the return travel rate $\omega^{-}$exhibits a critical transition and vanishes for return rates exceeding a critical value. The function $\rho\left(\omega^{-}\right)$is shown for a 1-d lattices system with 100 sites with $N$ agents/site. Insets: (a) $\rho\left(\omega^{-}\right)$for a scale-free network $(\gamma=1.5)$ of $10^{3}$ nodes populated uniformly with $\langle N\rangle=250, k_{\min }=5$ and $k_{\max }=50$; (b) $\rho\left(\omega^{-}\right.$) for an Erdős-Rényi network with 500 nodes and $\langle k\rangle=10$. Results were averaged over 50 realizations. Global travel rate was kept constant at $\omega=1$. Epidemic parameters are $\alpha=1, \beta=0.1$. Right: The attack ration as a function of both travel rate parameters $\omega^{ \pm}$for a lattice system. Solid lines represent constant global rate $\omega$ curves with logarithmic spacing. The total flux increases from bottom left to top right. Green regions corresponds to sustained outbreaks, red reflects the extinction regime. Epidemic rates, lattice and averaging parameters are the same as in the left panel.

location $j_{1}(t)=1 /\left(1+a e^{-\alpha t}\right)$ with $a=1-j_{1}(0) / j_{1}(0)$ yields

$$
j_{2}(t)=e^{\alpha t} \omega\left(\ln \frac{1+a e^{-\alpha t}}{1+a}+\ln e^{\alpha t}\right) .
$$

As $t \rightarrow \infty$ we have $\ln \left(1+a e^{-\alpha t}\right) \approx 0$, and thus $j_{2}(t) \sim \omega e^{\alpha t}$. The time lag between outbreaks in both populations is given by $\Delta \tau(q)=\tau_{2}(q)-\tau_{1}(q)$, where $\tau_{1}$ and $\tau_{2}$ are times when the concentrations of infecteds attains some threshold $q$ in the first and second location respectively. It follows that

$$
\Delta \tau(q) \sim \ln \frac{q}{\alpha \omega}-\ln \frac{q}{1-q}-\ln a,
$$

and for $q=1 / 2$ we obtain for the velocity $c \sim \Delta \tau^{-1}$ and thus

$$
c \sim\left(-\ln \frac{\alpha \omega}{2}-\ln a\right)^{-1} .
$$

This expression reproduces the slow convergence of the front velocity towards zero with decreasing travel rate which is in agreement with the results obtained by numerical solution of the mean-field problem. 

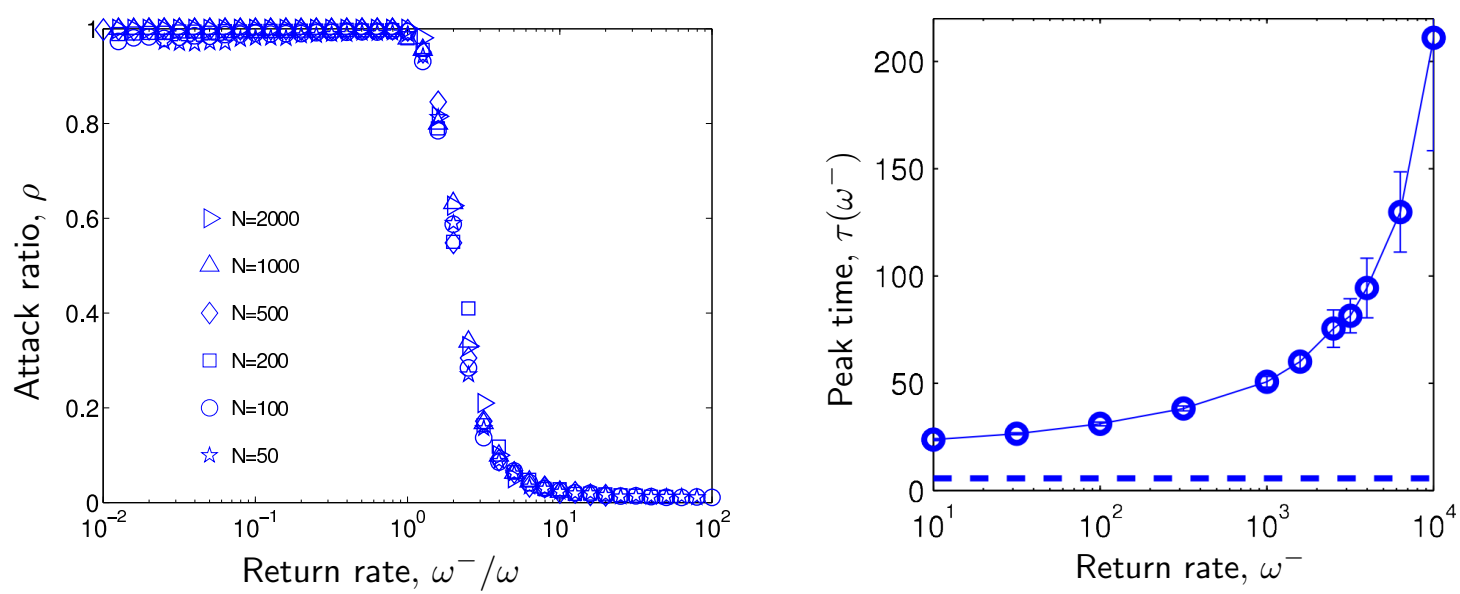

Figure 6: Effects in stochastic system: Left: The behavior of the attack ratio $\rho$ as a function of the ratio of return rate and $\omega^{-}$global rate $\omega$. Other parameters are identical to those of the 1-d system in Fig. (5). Right: Time lag $\tau\left(\omega^{-}\right)$as a function of $\omega^{-}$for the natural mobility model (circles) and ordinary reaction-diffusion (dashed line) on a scale-free network (with scaling exponent $\gamma=1.5$ and $10^{3}$ nodes with an average of $\langle N\rangle=250$ individuals per site. The curves depiced averages over 50 stochastic realizations.

\section{B. Front shape}

Front velocity is tightly connected with the shape of the front at the leading edge. In Fig. 2 front shapes as obtained from numerical solutions for both reaction-diffusion and natural mobility SIR models are shown. We observe that the reaction-diffusion slope is much more strongly affected by the global travel rate $\omega$. This is in contrast to the system with natural mobility patterns in which, just like the front velocity, the front shape converges to a fixed state as $\omega$ increases. The particular front shape is related to Kendall waves that have been empirically observed [23].

\section{Invasion Thresholds in natural human mobility models}

In addition to questions concerning the velocity of disease propagation, it is of fundamental importance to assess the conditions under which an epidemic propagates at all. Usually such a condition takes the form of a threshold in a system parameter. Thea most prominent example is the basic reproduction number, given by $R_{0}=\alpha / \beta$ for a SIR model. It quantifies the a number of secondary cases caused by a single infected individual in a totally susceptible population [1]. If $R_{0}>1$ an outbreak occurs, otherwise the epidemic wanes. Another threshold parameter in the metapopulation reaction-diffusion framework is the global invasion threshold. It represents the minimal required flux of individuals traveling between two locations [11] in order for a disease to propagate spatially. 
One of the most striking properties of the natural mobility model of Eqs. (7) is the existence of a novel type of threshold that is only determined by the return rate $\omega^{-}$, or equivalently by the typical time an individual spends at a distant location. The existence of this threshold is evident from Fig.5 that depicts the attack ratio $\rho$ (the total fraction of infecteds during an epidemic) as a function of the return rate $\omega^{-}$ on a) a one-dimensional lattice, b) and Erdős-Rényi network and c) an uncorrelated scale-free network. For low return rates the attack ratio is close to unity, as expected, a global outbreak occurs. However, with growing values of the return rate, the attack ratio drops almost to zero, i.e. no global outbreak occurs. The regime of high return rates corresponds to small dwelling times at distant locations. This implies that an infected does not have sufficient time to transfer the disease to susceptibles in unaffected locations before returning home. This effect is absent in reaction-diffusion systems. This novel type of threshold is a direct consequence of the properties of natural human mobility.

To assess the mutual impact of all travel parameters, i.e. the forward and return travel rates as well as the total flux on the dynamics we calculated the attack ratio for various parameter values on a homogeneous lattice. The results are presented in Fig. 5 . Note that our model exhibits the global $\omega$-limited invasion threshold that is also present in ordinary reaction-diffusion systems. However, for large total flux $\omega$, the system exhibits a global outbreak only if the return rate $\omega^{-}$is sufficiently small. Increasing the return rate the system enters a region that lacks a global outbreak. Consequently, only the return rates $\omega^{-}$is a limiting factor. This novel threshold can be estimated analytically. In the same spirit as introduced recently [18, 22] we find the following threshold relation:

$$
\frac{N \omega}{\beta+\omega^{-}}\left(R_{0}-1\right)>1
$$

The inverse of the sum of return rate $\omega^{-}$and recovery rate $\beta$ provides the typical time an infected individual spends on a distant location in the infected status. Using the relation $\omega=2 \omega^{+} \omega^{-} /\left(2 \omega^{+}+\omega^{-}\right)$ (compare (14)), we can write explicitly

$$
\frac{\omega^{-}}{\beta}<\frac{\omega^{+}}{\beta} 2 N\left(R_{0}-1\right)-1
$$

Note, that from [20] the empirically observed scaling $\rho=\rho\left(N \omega / \omega^{-}\right)$follows, see Fig. 5 and 6 . The figures exhibit the expected collapse of the data according to this scaling. The pronounced difference between ordinary reaction-diffusion systems and the natural mobility model is also captured in Fig. 6 that illustrates the epidemic peak time $\tau=\int I(t) t d t / \int I(t) d t$ on the return rate. The figure shows that by varying the return rate one can substantially increase the peak time in the natural mobility model, in contrast to the reaction-diffusion model. 

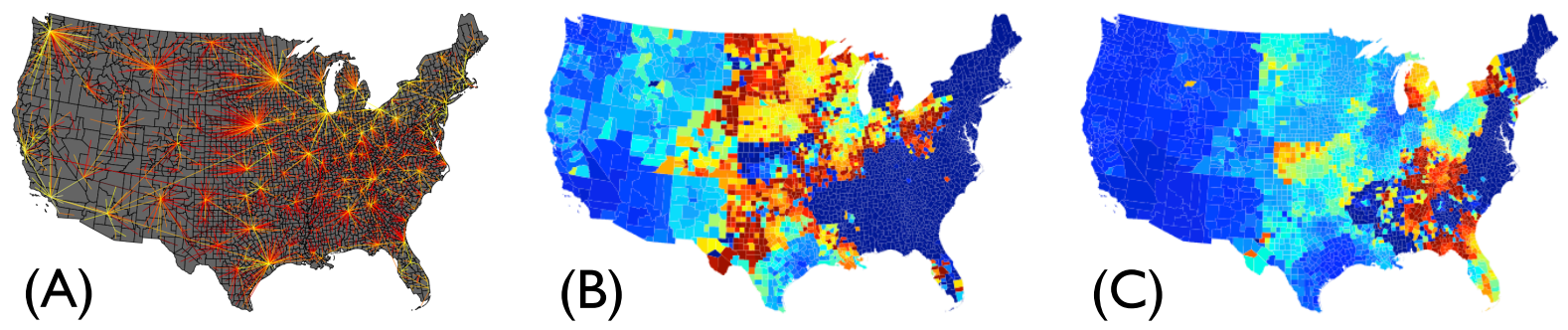

Figure 7: Natural human mobility and disease dynamics in realistic scenarios. (A): The minimal spanning tree of a multiscale mobility network in the United States. This network represents the skeleton of the most important transportation routes in a more complete network [4 24]. (B), (C): Comparison of the evolution of the SIR epidemic with initial outbreak in Los Angeles for the natural mobility model (B) and ordinary reaction-diffusion model (C). Snapshot of the number of infecteds after approximately 4 weeks. Color encode from high (red) to low (blue) the relative number of infecteds per county.

\section{DISCUSSION}

An unprecedented amount of information on human mobility available today requires adequate models that correctly capture the key features of natural human mobility in order to understand, describe and predict the dynamics of human mediated contagion phenomena. In the present work we pursued this goal and formulated an approach that can account for important features of natural human mobility that are intuitive and observed empirically. The model is based on a metapopulation approach assuming well-mixed local populations and explicit incorporation of individual mobility patterns conditional on base or home location. We considered regular bi-directional movements of the host between base locations and accessible destinations, systematically analyzed the model and compared it to established modeling approaches, e.g. effective force of infection and reaction-diffusion systems. We demonstrated that the latter are limiting cases of the natural human mobility model at low and high travel rates, respectively. For a regular lattice we derived a generalization of the Fisher-Kolmogorov equation and found that contrary to the reaction-diffusion approach, the front velocity of the epidemic does not increase unboundedly with increasing global travel rate, but saturates at a maximum level.

Although results for lattice and artificial random network topologies are extremely helpful in gaining fundamental insight into the dynamics and consequences of natural human mobility patterns on the patterns of disease spread, they at best mimic real world scenarios. A future task will be to investigate to what level these novel effects prevail in more realistic settings, i.e. real world mobility networks on which individuals move and transmit disease. We hypothesize that effects that are so dominant in par- 
simonious systems of the type described by the model of Eqs. 7 will also be present in more complex settings. Evidence for this has recently been revealed in a multiscale metapopulation system [19]. In order to illustrate the pronounced difference in disease dynamic patterns that are generated by ordinary reaction diffusion models on one hand and natural human mobility models on the other, we simulated both systems on the backbone of a realistic, multiscale mobility network in a real geographic setting. Fig. 7 illustrates snapshots of the timecourse of disease spread generated by both models. Nodes in the network are approx. 3000 counties in continental United States and links between them resemble the traffic flux. We observe a significantly smaller spreading speed in the natural mobility scenario compared with the reaction-diffusion model. This implies that estimates of spreading speeds, also in these more realistic setting, could have been overestimated in the past by models that rely on ordinary diffusion as a dispersal mechanism.

Although the study of natural human mobility on disease dynamics and related human mediated contagion processes requires more attention in future investigations, the results presented here as well as in our previous work [14, 18] will serve as a useful guide for developing more reliable large scale computational models for disease dynamics. The substantial differences of ordinary reaction-diffusion dynamics and the novel type of natural human mobility disperal suggest that disperal mechanism are among the most important modeling ingredients that require particular care when implemented in large scale computational models that are designed to make quantitative forecasts.

D.B. and V.B. acknowledge support from the Volkswagen Foundation. D.B. acknowledge support from EU-FP7 grant Epiwork.

[1] R.M. Anderson, R.M. May, Infectious Diseases of Humans (Oxford University Press, 1991)

[2] L. Hufnagel, D. Brockmann, T. Geisel, Proc. Natl. Acad. Sci. USA 101, 15124 (2004)

[3] C. Fraser, C.A. Donnelly, S. Cauchemez, W.P. Hanage, M.D. Van Kerkhove, T.D. Hollingsworth, J. Griffin, R.F. Baggaley, H.E. Jenkins, E.J. Lyons et al., Science 324(5934), 1557 (2009)

[4] D. Brockmann, L. Hufnagel, T. Geisel, Nature (London) 439, 462 (2006)

[5] M.C. González, C.A. Hidalgo, A.L. Barabási, Nature (London) 453, 779 (2008)

[6] S. Riley, Science 316, 1298 (2007)

[7] D. Brockmann, L. Hufnagel, Phys. Rev. Lett. 98, 178301 (2007)

[8] A. Kolmogorov, I. Petrovsky, N. Piscounov, Bull. de l'univ. d'état à Moscou, Sér. internat., sect. A 1, 1 (1937)

[9] R.A. Fisher, Ann. Eugenics 7, 355 (1937)

[10] L.A. Rvachev, I.M. Longini, Math. Biosc. 75, 3 (1985)

[11] V. Colizza, R. Pastor-Satorras, A. Vespignani, Nature Physics 3(4), 276 (2007) 
[12] S. Rushton, A.J. Mautner, Biometrika 42, 126 (1955)

[13] T.J. Hagernaas, C.A. Donnelly, N.M. Ferguson, J. Theor. Biol. 229, 349 (2004)

[14] V.V. Belik, T. Geisel, D. Brockmann, in Proceedings of 2009 International Conference on Computational Science and Engineering (IEEE, 2009), pp. 932-935, ISBN 978-1-4244-5334-4

[15] D. Brockmann, Human Mobility and Spatial Disease Dynamics, in Reviews of Nonlinear Dynamics and Complexity (Wiley-VCH, 2009), pp. 1-24

[16] C. Song, Z. Qu, N. Blumm, A.L. Barabási, Science 327, 1018 (2010)

[17] C. Song, T. Koren, P. Wang et al., Nat. Phys. 6, 818 (2010)

[18] V. Belik, T. Geisel, D. Brockmann, Phys. Rev. X (2011), in press.

[19] D. Balcan, A. Vespignani, Nature Physics (2011)

[20] L. Sattenspiel, K. Dietz, Math. Biosc. 128, 71 (1995)

[21] M.J. Keeling, P. Rohani, Ecol. Lett. 5, 20 (2005)

[22] V. Colizza, A. Vespignani, Phys. Rev. Lett. 99, 148701 (2007)

[23] U. Naether, E.B. Postnikov, I.M. Sokolov, Eur. Phys. J. B 65, 353 (2008)

[24] C. Thiemann, F. Theis, D. Grady, R. Brune, D. Brockmann, PLoS ONE 5(11), e15422 (2010) 